\title{
An Optimisation on the Grassmannian with Applications to Quantum Chemistry
}

\author{
Yuri Alexandre Aoto ${ }^{1}$ \\ Centro de Matemática, Computação e Cognição/UFABC, Santo André - SP \\ Márcio Fabiano da Silva ${ }^{2}$ \\ Centro de Matemática, Computação e Cognição/UFABC, Santo André - SP
}

\begin{abstract}
In this work we propose an algorithm to find critical points of the inner product between an element of the Grassmannian and a fixed point of the projective space of the exterior algebra where the Grassmannian is embedded. This has interesting applications to electronic structure theory, where the wave functions are represented by elements of the exterior algebra. This method is exemplified for a Grassmannian that is a model for the hydrogen molecule, $\mathrm{H}_{2}$.
\end{abstract}

Keywords. Grassmannian, differential geometry, exterior algebra, quantum chemistry

\section{Introduction and motivation}

In the field of Quantum Chemistry, and in particular in electronic structure theory, one is interested on studying the wave function associated to the electrons. All the chemical and physical properties of atoms and molecules are to some extent related to the behaviour of their electrons, and thus a precise characterisation of their wave function is central in modern studies in computational chemistry (see [5], for example). From the rules of quantum mechanics, the wave functions form a vector space and must be anti-symmetric with respect to the exchange of any two electrons, see Chapter XIV of [7]. As shown in [8,9], this is accomplished by representing the wave functions as elements of the exterior algebra, $\bigwedge^{n} \mathcal{V}$, of another vector space $\mathcal{V}$, with $n$ being the number of electrons in the system. $\mathcal{V}$ is conveniently chosen to represent as best as possible the region around the atoms where the electrons are, and its elements are called spin orbitals in this context.

The calculation of the wave function is a difficult task. Although a number of procedures and approximations exist, with several implementations in computational packages, the development of new methods, as well as the study of the performance and improvement of existing ones, is an active field of research, see [6] for example. In particular, the clear understanding of the underlying mathematical framework of these methods can be an important guide to the development of more sophisticated and efficient procedures. With this motivation, in this work we adapt an optimisation procedure at the Grassmannian, described in [1], for applications in computational chemistry, with the main goal of clarifying how the geometry of this manifold is related to the quality of the approximations used in this field of research. We will start by recalling some basic facts and definitions about the Grassmannian, presenting the algorithm that we will use as starting point; In Section 3 the problem we propose to solve is stated, followed by the equations we have developed, in Section 4; The equations and the problem are put in a general and straightforward form and thus can be used for applications other than in electronic structure theory. At the end, an example is presented and the connection field of Quantum Chemistry is discussed.

\footnotetext{
1yuri.aoto@ufabc.edu.br

2 marcio.silva@ufabc.edu.br
} 


\section{Basics: the Grassmannian and the Plücker embedding}

Given a $K$-dimensional real vector space $\mathcal{V}$, its Grassmannian or Grassmann manifold of order $n \leq K$ is denoted by $\operatorname{Gr}(n, \mathcal{V})$, and is defined as the set of all $n$-dimensional vector subspaces of $\mathcal{V}$. See for example Chapter 1 of [4] for the definition of Grassmannians. Furthermore, the $n$-th exterior power of $\mathcal{V}$ is denoted by $\bigwedge^{n} \mathcal{V}$, and it is composed by all anti-symmetric (also known as skew-symmetric) tensors of order $n$ on $\mathcal{V}$, see Chapter 8 of [3]. This is an algebra under the exterior product, $\wedge$. Given a basis for $\mathcal{V}$, say $\left\{u_{1}, u_{2}, \ldots, u_{K}\right\}$, the set formed by all exterior products of these elements, taken $n$ by $n$ without repetition, is a basis for $\bigwedge^{n} \mathcal{V}$ :

$$
\left\{u_{I_{1}} \wedge u_{I_{2}} \wedge \cdots \wedge u_{I_{n}}\right\}=\left\{\Phi_{I}\right\}
$$

where $I$ is a multi-index, running over all possible subsets of $\{1,2, \ldots, K\}$ of size $n$. Thus, an arbitrary element of $\bigwedge^{n} \mathcal{V}$ is given by:

$$
\Psi=\sum_{I} c_{I} u_{I_{1}} \wedge u_{I_{2}} \wedge \cdots \wedge u_{I_{n}}=\sum_{I} c_{I} \Phi_{I}, \text { with } c_{I} \in \mathbb{R} .
$$

Some elements of $\bigwedge^{n} \mathcal{V}$ can be written simply as $\Phi=v_{1} \wedge v_{2} \wedge \cdots \wedge v_{n}$, with $v_{i} \in \mathcal{V}$ (for instance, each element of the basis in Equation (1)). However, this does not hold for all elements of $\bigwedge^{n} \mathcal{V}$, and the elements that can be put in this simpler form are said to be decomposable. If $\left\{v_{i}\right\}_{i=1}^{n}$ is linearly independent, it spans a $n$-dimensional subspace of $\mathcal{V}$, say $[\Phi] \in \operatorname{Gr}(n, \mathcal{V})$; furthermore, if $\left\{v_{i}^{\prime}\right\}_{i=1}^{n}$ is another basis for $[\Phi]$, the decomposable element formed by these elements is proportional to the first:

$$
v_{1}^{\prime} \wedge v_{2}^{\prime} \wedge \cdots \wedge v_{n}^{\prime}=\lambda v_{1} \wedge v_{2} \wedge \cdots \wedge v_{n} \quad \lambda \in \mathbb{R}, \lambda \neq 0 .
$$

This is an one-to-one correspondence between the $n$-dimensional vector subspaces of $\mathcal{V}$ and the equivalence classes of decomposable elements of $\bigwedge^{n} \mathcal{V}$ (under multiplication by non-zero elements of $\mathbb{R})$, that induces a natural embedding of the $\operatorname{Grassmannian} \operatorname{Gr}(n, \mathcal{V})$ in the projective space of $\bigwedge^{n} \mathcal{V}$, the Plücker embedding (see, for example, Chapter 1, Section 5, of [4]):

$$
\begin{aligned}
\operatorname{Gr}(n, \mathcal{V}) & \hookrightarrow \mathbb{P} \bigwedge^{n} \mathcal{V} \\
{[\Phi] } & \mapsto\left[v_{1} \wedge v_{2} \wedge \cdots \wedge v_{n}\right]
\end{aligned}
$$

with $\left\{v_{i}\right\}_{i=1}^{n}$ being any basis for the vector space $[\Phi]$. For the sake of a simpler notation, we will not distinguish here between the Grassmannian and its image under this embedding. We will focus on finite dimensional vector spaces over the field of real numbers, but extensions of these definitions to infinite dimensional vector spaces, and over other fields, are possible.

\subsection{The Newton-Grassmann method of Absil and coworkers}

Let $\mathcal{V}$ be a vector space of dimension $K$, and $\mathcal{B}$ a fixed basis for it. Given $\Phi \in \operatorname{Gr}(n, \mathcal{V})$, let $Y$ be the matrix whose columns contain the coefficients of a basis of $\Phi$ on the basis of $\mathcal{V}$. This is an element of the non-compact Stiefel manifold, $\mathrm{ST}(n, K)$, defined as the set of all $K \times n$ matrices of rank $n$. Let $f: \operatorname{Gr}(n, \mathcal{V}) \rightarrow \mathbb{R}$ be a function defined on the Grassmannian with real values, sufficiently smooth, one wants to optimise. Let

$$
\begin{aligned}
f_{\diamond}: \mathrm{ST}(n, K) & \rightarrow \mathbb{R} \\
Y & \mapsto f(\operatorname{span}(Y)),
\end{aligned}
$$

be the corresponding function on the Stiefel manifold, where $\operatorname{span}(Y)$ is the column space of $Y$, that is identified with an element of $\operatorname{Gr}(n, \mathcal{V})$, by the canonical bijection between $\mathcal{V}$ and $\mathbb{R}^{K}$. The 
calculation is done now in the Stiefel manifold and, according to the method proposed by Absil and coworkers in [1]:

- One first solves the following equation for the unknown $\eta_{\diamond Y} \in H_{Y}=\left\{Y_{\perp} K: K \in \mathbb{R}^{(K-n) \times n}\right\}$ :

$$
\Pi_{Y_{\perp}} D\left(\Pi_{\perp} \operatorname{grad} f_{\diamond}(\cdot)\right)(Y)\left[\eta_{\diamond Y}\right]=-\Pi_{Y_{\perp}} \operatorname{grad} f_{\diamond}(Y)
$$

- And then update $\Phi \rightarrow \Phi_{\text {new }}$ by moving along the geodesic on the Grassmannian in the direction of $\operatorname{span}\left(\eta_{\diamond Y}\right)$, by computing a singular value decomposition of $\eta_{\diamond Y}=U \Sigma V^{T}$ and calculating:

$$
\Phi_{\text {new }}=\operatorname{span}(Y V \cos \Sigma+U \sin \Sigma) .
$$

In these equations, the gradient of $f_{\diamond}$ at $Y$ is the $K \times n$ matrix whose entries are given by $\left(\operatorname{grad} f_{\diamond}(Y)\right)_{q}^{p}=\frac{\partial f_{\diamond}(Y)}{\partial Y_{q}^{p}}(Y) ; D F(x)[y]=\left.\frac{d}{d t} F(x+t y)\right|_{t=0}$ is the directional derivative of $F$ at $x$ in the direction of $y$; and $\Pi_{W_{\perp}}=I-W\left(W^{T} W\right)^{-1} W^{T}$ is the projection onto the orthogonal complement of the matrix $W$.

\section{The problem}

Given a $K$-dimensional vector space $\mathcal{V}$, with an inner product $(\cdot, \cdot)$, let $\mathbb{P} \wedge^{n} \mathcal{V}$ be the projective space of the $n$-th exterior power of $\mathcal{V}(n \leq K)$, and let $\Psi \in \mathbb{P} \bigwedge^{n} \mathcal{V}$ be a fixed, but arbitrary, point of this space, taken as the reference point in the following. In general, $\Psi \notin \operatorname{Gr}(n, \mathcal{V}) \subset \mathbb{P} \bigwedge^{n} \mathcal{V}$. How to find the stationary points of the function

$$
f(\Phi)=\frac{\langle\Phi, \Psi\rangle}{\sqrt{\langle\Phi, \Phi\rangle\langle\Psi, \Psi\rangle}}
$$

in $\operatorname{Gr}(n, \mathcal{V}) \subset \mathbb{P} \bigwedge^{n} \mathcal{V}$, where $\langle\cdot, \cdot\rangle$ is the inner product at $\bigwedge^{n} \mathcal{V}$, induced by $(\cdot, \cdot)$ ?

\section{Solving the proposed problem by Newton-Grassmann}

To solve the problem suggested in Section 3, we will use the algorithm proposed by Absil and coworkers, described in Section 2.1. The update step $\Phi \rightarrow \Phi_{\text {new }}$ does not depend on the function to be optimised, and it is of straightforward implementation. However, it is not obvious how to obtain Equation (7), and this is the central objective in the present work. In the following, we will assume a fixed orthonormal basis for the vector space $\mathcal{V}$, and the induced basis for the exterior algebra $\bigwedge^{n} \mathcal{V}$

First of all, given $\Psi$ as in Equation (2), with unit norm, and $\Phi=\operatorname{span}(Y)$, with $Y \in \operatorname{ST}(n, K)$ the function $f$ is calculated as

$$
f(\Phi)=f_{\diamond}(Y)=\frac{1}{\sqrt{\operatorname{det}\left(Y^{T} Y\right)}} \sum_{I} c_{I} \operatorname{det}\left(\left.Y\right|_{I}\right)=\frac{1}{\sqrt{\operatorname{det}\left(Y^{T} Y\right)}} \sum_{I} c_{I} F_{I} .
$$

Note that the term within the square root guarantees that the expression is independent on the choice of $Y \in \mathrm{ST}(n, K)$ used to represent the vector space $\Phi$. If $Y$ forms an orthonormal basis for $\mathcal{V}$, such term equals one. Furthermore, since $\Phi$ is assumed to have dimension $n$, such term is never zero. Although one can easily generate an orthonormal basis for $\Phi$ before calculating $f_{\diamond}$, we note that $f_{\diamond}(Y)=f(\operatorname{span}(Y))$ must hold for all $Y$ in the Stiefel manifold, and the normalisation factor is important to consider when deriving Equation (7) for the present function $f$, even if a normalised $Y$ is used during actual computation. 
The notation $\left.Y\right|_{I}$ stands for the submatrix of $Y$ formed with the rows that are in the multi-index I. For example, for

$$
Y=\left(\begin{array}{ccc}
Y_{1}^{1} & Y_{2}^{1} & Y_{3}^{1} \\
Y_{1}^{2} & Y_{2}^{2} & Y_{3}^{2} \\
Y_{1}^{3} & Y_{2}^{3} & Y_{3}^{3} \\
Y_{1}^{4} & Y_{2}^{4} & Y_{3}^{4} \\
Y_{1}^{5} & Y_{2}^{5} & Y_{3}^{5}
\end{array}\right), \text { we have }\left\{\begin{array}{c}
\left.Y\right|_{I=\{1,2,3\}}=\left(\begin{array}{ccc}
Y_{1}^{1} & Y_{2}^{1} & Y_{3}^{1} \\
Y_{1}^{2} & Y_{2}^{2} & Y_{3}^{2} \\
Y_{1}^{3} & Y_{2}^{3} & Y_{3}^{3}
\end{array}\right) \\
\left.Y\right|_{I=\{1,4,5\}}=\left(\begin{array}{ccc}
Y_{1}^{1} & Y_{2}^{1} & Y_{3}^{1} \\
Y_{1}^{4} & Y_{2}^{4} & Y_{3}^{4} \\
Y_{1}^{5} & Y_{2}^{5} & Y_{3}^{5}
\end{array}\right),
\end{array}\right.
$$

and so on. Thus, Equation (10) says that, after taking the normalisation into account, $f_{\diamond}$ is calculated by summing the contribution of all terms of the reference point $\Psi$, this contribution being the corresponding coefficient times the determinant of $\left.Y\right|_{I}, F_{I}=\operatorname{det}\left(\left.Y\right|_{I}\right)$.

Now, Equation (7) is rewritten for the present case as the following system of linear equations:

$$
\sum_{r=1}^{K} \sum_{s=1}^{n} \mathbf{X}_{q s}^{p r}\left(\eta_{\diamond Y}\right)_{s}^{r}=\mathbf{C}_{q}^{p} \quad \begin{aligned}
& p=1, \ldots, K \\
& q=1, \ldots, n
\end{aligned}
$$

with

$$
\begin{gathered}
\mathbf{C}_{q}^{p}=\sum_{I} c_{I}\left(G_{I}-F_{I} Y\right)_{q}^{p}=\left(\Pi_{Y_{\perp}}\right)_{\bar{p}}^{p} \sum_{I} c_{I}\left(G_{I}\right)_{q}^{\bar{p}} \text {, and } \\
\mathbf{X}_{q s}^{p r}=\sum_{I} c_{I}\left\{\left(G_{I}-F_{I} Y\right)_{q}^{p}(Y)_{s}^{r}-\left(\Pi_{Y_{\perp}}\right)_{\bar{p}}^{p}\left(\tilde{H}_{I}\right)_{q s}^{\bar{p} r}\right\}=\left(\Pi_{Y_{\perp}}\right)_{\bar{p}}^{p} \sum_{I} c_{I}\left(\left(G_{I}\right)_{q}^{\bar{p}}(Y)_{s}^{r}-\left(\tilde{H}_{I}\right)_{q s}^{\bar{p} r}\right) .
\end{gathered}
$$

The quantities $G_{I}$ and $\tilde{H}_{I}$ are defined as:

$$
\begin{gathered}
\left(G_{I}\right)_{q}^{p}=\left.\operatorname{det}\left(Y \stackrel{q}{\leftarrow} e_{p}\right)\right|_{I} \\
\left(H_{I}\right)_{q s}^{p r}=\left.\operatorname{det}\left(Y \stackrel{q}{\leftarrow} e_{p} \stackrel{s}{\leftarrow} e_{r}\right)\right|_{I} \quad ; \quad\left(\tilde{H}_{I}\right)_{q s}^{p r}=\left\{\begin{array}{ll}
\left(H_{I}\right)_{q s}^{p r} & \text { if } s \neq q \\
-F_{I} \delta_{p r} & \text { otherwise }
\end{array} .\right.
\end{gathered}
$$

The notation $A \stackrel{q}{\leftarrow} b$ represents the matrix $A$ with the $q$-th column replaced by $b$, whereas $e_{p}$ is the $p$-th element of the canonical basis of $\mathbb{R}^{K}$. For example, given the matrix $Y$ of Equation (11):

$$
\begin{gathered}
\left(G_{I=\{1,2,3\}}\right)_{3}^{3}=\operatorname{det}\left(\begin{array}{ccc}
Y_{1}^{1} & Y_{2}^{1} & 0 \\
Y_{1}^{2} & Y_{2}^{2} & 0 \\
Y_{1}^{3} & Y_{2}^{3} & 1
\end{array}\right)=Y_{1}^{1} Y_{2}^{2}-Y_{1}^{2} Y_{2}^{1} ; \\
\left(G_{I=\{1,2,3\}}\right)_{2}^{4}=\operatorname{det}\left(\begin{array}{ccc}
Y_{1}^{1} & 0 & Y_{3}^{1} \\
Y_{1}^{2} & 0 & Y_{3}^{2} \\
Y_{1}^{3} & 0 & Y_{3}^{3}
\end{array}\right)=0 ; \\
\left(H_{I=\{1,4,5\}}\right)_{12}^{15}=\operatorname{det}\left(\begin{array}{ccc}
1 & 0 & Y_{3}^{1} \\
0 & 0 & Y_{3}^{4} \\
0 & 1 & Y_{3}^{5}
\end{array}\right)=-Y_{3}^{4} .
\end{gathered}
$$

The above equations are valid for an orthonormal basis of $\Phi$ (note, for instance, the absence of normalisation factors as we have for $f$, Equation (10)). Furthermore, the matrix $\eta_{\diamond Y}$ must be in the space $H_{Y}=\left\{Y_{\perp} K: K \in \mathbb{R}^{(K-n) \times n}\right\}$, that is, its columns must be orthogonal to the columns of $Y$. This is guaranteed by extending the matrices $\mathbf{X}$ and $\mathbf{C}$ (with the columns of $Y$ and zeros, respectively) to impose this orthogonality condition automatically when solving Equation (12). 
Although Equation (10) is simply obtained using the tools of exterior algebra, where the determinant plays a central role, substitution of Equation (10) in Equation (7) leads to a lengthy derivation. To see how the quantities $G_{I}$ and $H_{I}$ appear, note that:

$$
\begin{aligned}
\frac{\partial \operatorname{det}\left(\left.Y\right|_{I}\right)}{\partial Y_{q}^{p}}(Y) & =\operatorname{tr}\left(\operatorname{adj}\left(\left.Y\right|_{I}\right) \frac{\partial\left(\left.Y\right|_{I}\right)}{\partial Y_{q}^{p}}(Y)\right) \\
& =\operatorname{tr}\left(\begin{array}{ccc}
\left.\operatorname{det}\left(Y \stackrel{1}{\leftarrow} \delta_{1 q} e_{p}\right)\right|_{I} & \ldots & \left.\operatorname{det}\left(Y \stackrel{1}{\leftarrow} \delta_{n q} e_{p}\right)\right|_{I} \\
\vdots & \ddots & \vdots \\
\left.\operatorname{det}\left(Y \stackrel{n}{\leftarrow} \delta_{1 q} e_{p}\right)\right|_{I} & \ldots & \left.\operatorname{det}\left(Y \stackrel{n}{\leftarrow} \delta_{n q} e_{p}\right)\right|_{I}
\end{array}\right) \\
& =\left(G_{I}\right)_{q}^{p},
\end{aligned}
$$

where $\operatorname{adj}(W)$ represents the classical adjoint, or adjugate, of $W$. In Equation (20) the Jacobi formula for the derivative of the determinant has been used, whereas Equation (21) is obtained using the fact that the product between $\operatorname{adj}(W)$ and a column vector $b$ is the column vector with $\operatorname{det}(W \stackrel{p}{\leftarrow} b)$ in the $p$-th entry. The quantities $H_{I}$ appear similarly, from the directional derivative of the gradient in the left-hand side of Equation (7).

\section{Application to Quantum Chemistry}

Back to our motivation, the electronic wave functions of a $n$-electron system belong to $\bigwedge^{n} \mathcal{V}$. However, the extremely large dimension of $\Lambda^{n} \mathcal{V}$ to accurately represent the wave function makes its calculation and storage impractical for most of the cases. Thus, a number of procedures exist to truncate such expansion, keeping only the most important contributions to the actual wave function. See [5] for a detailed description of the main methods to approximate the wave function. One of the ways to do this is to use an element of $\operatorname{Gr}(n, \mathcal{V}) \subset \mathbb{P} \bigwedge^{n} \mathcal{V}$ as an approximate wave function: $\Phi=u_{1} \wedge u_{2} \wedge \cdots \wedge u_{n}$. From the chemical point of view, there is one electron associated to the spin orbital $u_{1}$, another associated to the spin orbital $u_{2}$, and so on. This is called orbital approximation and provides an interpretation of the wave function in terms of occupied orbitals and electronic configuration, central to chemistry. However, for such approximation be reasonable, $\Phi$ has to be optimised according to some criteria (that is, optimal orbitals $u_{i}$ must be used). In one of the most important methods in computational chemistry, the Hartree-Fock method (see Chapter 10 of [5]), $\Phi$ is optimised to minimise the energy of the wave function.

The Hartree-Fock method, however, very often fails to provide a qualitative description of the exact wave function [5], because it might not be close enough to the Grassmannian. In these cases more elaborate methods must be used, for instance as described in [6]. The main application of this work is to characterise when and why this happens, by obtaining the point of the Grassmannian closest to the exact wave function. Note that a metric in $\mathbb{P} \bigwedge^{n} \mathcal{V}$ can be introduced, among several ways, with $D\left(\Psi, \Psi^{\prime}\right)=\arccos \left|\left\langle\Psi, \Psi^{\prime}\right\rangle\right|$, where $\Psi$ and $\Psi^{\prime}$ are assumed normalised [2]. Thus, the distance from the exact wave function to the Grassmannian can be obtained by finding the maximum of the function $f$, Equation (9). Its knowledge and characterisation should help to understand when the Hartree-Fock method is accurate enough for the description of the electronic system. Aiming at this application, the equations presented in Section 4 have been implemented using Python. This implementation considers specific cases for the matrix $Y$ and reference point $\Psi$, e.g. the decomposition of $Y$ into alpha and beta spins. Test calculations have been carried out, showing that the algorithm can be used for reasonably large systems [2]. Further calculations over a broader set of molecules to test this method for our target application are in progress and are the subject of current research. 


\subsection{An example}

The smallest values for $n$ and $\operatorname{dim} \mathcal{V}$ for which the Grassmannian is not a projective space is $\operatorname{Gr}(2, \mathcal{V})$, with $\operatorname{dim} \mathcal{V}=4: \mathcal{V}=\operatorname{span}\left\{u_{1}, u_{2}, u_{3}, u_{4}\right\}$. This is a model for studying the hydrogen molecule, $\mathrm{H}_{2}$, that has two electrons. A common choice for this basis (see Section 5.2 of [5]) is to use $u_{1}$ and $u_{2}$ as the bonding spatial orbital, but with opposite spins, and $u_{3}$ and $u_{4}$ as the anti-bonding spatial orbital, again with opposite spins, forming an orthonormal basis. The second exterior power of $\mathcal{V}$ is:

$$
\bigwedge^{2} \mathcal{V}=\operatorname{span}\left\{u_{1} \wedge u_{2}, u_{1} \wedge u_{3}, u_{1} \wedge u_{4}, u_{2} \wedge u_{3}, u_{2} \wedge u_{4}, u_{3} \wedge u_{4}\right\}
$$

and an arbitrary element of this vector space is $\sum_{I} c_{I} \Phi_{I} \in \bigwedge^{2} \mathcal{V}$, with $c_{I} \in \mathbb{R}, \Phi_{i j}=u_{i} \wedge u_{j}$, and $i<j$. We will consider $\Psi=c_{12} \Phi_{12}+c_{34} \Phi_{34}$ as the reference point. Note that, for $\Psi \notin \operatorname{Gr}(2, \mathcal{V})$, for $c_{12} c_{34} \neq 0$, which follows directly from the Plücker relation for this Grassmannian, see for example Chapter 1, Section 5, of [4]. This element of $\bigwedge^{2} \mathcal{V}$ represents the exact ground state wave function of the hydrogen molecule, and it is commonly represented in Quantum Chemistry as:

$$
c_{12}\left(\begin{array}{l}
u_{3}-u_{4} \\
u_{1} \uparrow \downarrow u_{2}
\end{array}\right)+c_{34}\left(\begin{array}{l}
u_{3} \uparrow \downarrow u_{4} \\
u_{1} \longleftarrow u_{2}
\end{array}\right) .
$$

In this case, the symmetry and spin of the total system impose the remaining coefficients $c_{i j}$ to be zero.

Starting the procedure described in Section 4 at $\Phi=\left(a u_{1}-b u_{3}\right) \wedge\left(a u_{2}+b u_{4}\right) \in \operatorname{Gr}(2, \mathcal{V})$, the important quantities we need are:

$$
\begin{gathered}
Y=\left(\begin{array}{cc}
a & 0 \\
0 & a \\
-b & 0 \\
0 & b
\end{array}\right) ; \quad G_{I=\{1,2\}}=\left(\begin{array}{cc}
a & 0 \\
0 & a \\
0 & 0 \\
0 & 0
\end{array}\right) ; \quad G_{I=\{3,4\}}=\left(\begin{array}{cc}
0 & 0 \\
0 & 0 \\
b & 0 \\
0 & -b
\end{array}\right) ; \\
F_{I=\{1,2\}}=a^{2} ; \quad F_{I=\{3,4\}}=-b^{2} ; \\
\left(H_{I=\{1,2\}}\right)_{q q}^{p p}=-a^{2} ;\left(H_{I=\{3,4\}}\right)_{q q}^{p p}=b^{2} \quad \text { for all } p, q \\
\left(H_{I=\{1,2\}}\right)_{12}^{12}=\left(H_{I=\{1,2\}}\right)_{21}^{21}=\left(H_{I=\{3,4\}}\right)_{12}^{34}=\left(H_{I=\{3,4\}}\right)_{21}^{43}=1 ; \\
\left(H_{I=\{1,2\}}\right)_{21}^{12}=\left(H_{I=\{1,2\}}\right)_{12}^{21}=\left(H_{I=\{3,4\}}\right)_{21}^{34}=\left(H_{I=\{3,4\}}\right)_{12}^{43}=-1 ;
\end{gathered}
$$

and finally $\left(H_{I}\right)_{q s}^{p r}=0$ for the remaining cases. With these elements, the application of the equations of Section 4 is straightforward, and in Table 1 we give the convergence pattern of a specific case. Note that the optimisation converges fast to the vector space spanned by $\left\{u_{1}, u_{2}\right\}$, that is indeed a critical point of the function $f$, with $\left\langle u_{1} \wedge u_{2}, \Psi\right\rangle=c_{12}$. It will be the global maximum of the function $f$ whenever $\left|c_{12}\right|>\left|c_{34}\right|$, what is always the case for the wave function of the hydrogen molecule, irrespective of the inter-nuclear distance between the two hydrogen atoms. Two situations however arise: When $\left|c_{12}\right| \gg\left|c_{34}\right|$ the Hartree-Fock wave function, that minimises the energy at the Grassmannian, is also $\left\{u_{1}, u_{2}\right\}$ and it is a good approximation to the exact wave function (see Chapter 10 of [5]). This happens close to the equilibrium geometry of the molecule. When the atoms become far from each other, however, $\left|c_{12}\right| \approx\left|c_{34}\right|$ and the Hartree-Fock wave function does not represent the exact wave function qualitatively. It is not close to the maximum of the function $f$, that is always the vector space spanned by $\left\{u_{1}, u_{2}\right\}$, indicating that the maximum $f$ can be used to assess the quality of the Hartree-Fock wave function. 
Table 1: Application of the algorithm for the Grassmannian $\operatorname{Gr}(2, \mathcal{V}), \operatorname{dim} \mathcal{V}=4$, as outlined above, with $c_{12}=0.8, c_{34}=-0.6, a=0.9701425$, and $b=0.24253563$.

\begin{tabular}{cccc}
\hline iteration & $\Phi \in \operatorname{Gr}(2, \mathcal{V})$ & $f(\Phi)$ & $|\mathbf{C}|_{2}$ \\
\hline 0 & $\left(0.97014 u_{1}-0.24254 u_{3}\right) \wedge\left(0.97014 u_{2}+0.24254 u_{4}\right)$ & 0.717647 & 0.465859 \\
1 & $\left(0.99976 u_{1}+0.02169 u_{3}\right) \wedge\left(0.99976 u_{2}-0.02169 u_{4}\right)$ & 0.799342 & 0.042927 \\
2 & $\left(1.0 u_{1}-0.00001 u_{3}\right) \wedge\left(1.0 u_{2}+0.00001 u_{4}\right)$ & 0.7999999997 & $2.695010^{-5}$ \\
\hline
\end{tabular}

\section{Concluding remarks}

We present an optimisation procedure at the Grassmannian $\operatorname{Gr}(n, \mathcal{V})$, to find the critical points of the inner product with an arbitrary element of the projective space of the exterior algebra $\mathbb{P} \wedge^{n} \mathcal{V}$. This has potential applications in Quantum Chemistry, as it is an algorithm that explores the intrinsic Riemannian geometry of the Grassmannian to perform an optimisation of the electronic wave function, and we think that this work can booster further applications of concepts of differential geometry to the field of Quantum Chemistry.

\section{Acknowledgements}

This research has been supported by grants \#2017/21199-0 and \#2018/04617-6, São Paulo Research Foundation (FAPESP).

\section{References}

[1] Absil, P.-A. and Mahony, R. and Sepulchre, R. Riemannian Geometry of Grassmann Manifolds with a View on Algorithmic Computation, Acta Appl. Math., 2004, 80, 199-220, DOI: 10.1023/B:ACAP.0000013855.14971.91.

[2] Aoto, Y. A and da Silva M. F. Calculating the distance from an electronic wave function to the manifold of Slater determinants through the geometry of Grassmannians, Phys. Rev. A, 2020, 102, 052803, DOI: 10.1103/PhysRevA.102.052803.

[3] Bowen, R. M. and Wand, C.-C. Introduction to Vectors 83 Tensors. Dover, 2008.

[4] Griffiths, P. and Harris, J. Principles of Algebraic Geometry. Wiley, 1978.

[5] Helgaker, T. and Jørgensen, P. and Olsen, J. Molecular Electronic Structure Theory. Wiley, 2000.

[6] Lischka H. et. al. Multireference Approaches for Excited States of Molecules, Chem. Rev., 2018, 118, 7293, DOI: 10.1021/acs.chemrev.8b00244.

[7] Messiah, A. Quantum Mechanics. Dover, 1999.

[8] Mundim, K. C Anticommuting space: an alternative formulation of the wavefunction antisymmetry description, J. Phys. France, 1989, 50, 11-17, DOI: 10.1051/jphys:0198900500101100.

[9] Mundim, K. C and Mundim, M. S. P. Álgebra de Grassmann e a Teoria Quântica, Revista Brasileira de Ensino de Física, 1997, 19, 209-233. 